\title{
Mieux comprendre l'impact des évènements extrêmes sur les littoraux nécessite davantage d'observations in situ
}

\section{Xavier BERTIN ${ }^{1}$, Kévin MARTINS ${ }^{2}$, Marc PEZERAT ${ }^{1}$, Baptiste MENGUAL ${ }^{1}$, Laura LAVAUD ${ }^{1}$, Thibault COULOMBIER ${ }^{1}$}

1. UMR 7266 LIENSs, CNRS-Université de La Rochelle, 2 rue Olympe de Gouges, 17000 La Rochelle.

xbertin@univ-Ir.fr

2. UMR 5805 EPOC, CNRS-Université de Bordeaux, Pessac, France

\section{Résumé :}

Les zones littorales abritent une part croissante de la population mondiale en dépit de l'augmentation des risques littoraux dans un contexte de changement climatique. Cependant, l'hydrodynamique des zones littorales en présence de vagues de tempête reste encore mal connue, principalement du fait de la rareté des observations in situ lors des évènements extrêmes. Dans cette étude, nous présentons une campagne de mesure réalisée au niveau d'une plage dissipative située au sud-ouest de l'Ile d'Oléron en présence de vagues de tempête. L'analyse de ces données et l'application d'un modèle 1D de conservation du flux d'énergie des vagues montrent tout d'abord que les vagues de tempête subissent une dissipation bien moindre que ce qui est prédit par les principaux modèles de déferlement bathymétrique en utilisant des paramètres par défaut. Nos données montrent ensuite que des ondes infragravitaires (IG) à très basse fréquence $(f=$ $0.005 \mathrm{~Hz}$ ) peuvent se développer le long du rivage en présence de zones de déferlement kilométriques. L'application du système de modélisation XBeach suggère que ces ondes très longues résultent en partie de l'appariement d'ondes IG libres et liées, se propageant avec des célérités légèrement différentes sur de grandes distances. A travers ces deux exemples, notre étude suggère qu'une meilleure compréhension et prédiction des aléas littoraux implique l'acquisition de nouvelles mesures in situ dans les zones littorales en présence de vagues de tempête.

\section{Mots-clés :}

Vagues, Déferlement, Ondes infra-gravitaires, Plage dissipative, Transferts d'énergie, Ile d'Oléron.

\section{Introduction}

Les zones littorales abritent une part croissante de la population mondiale, en dépit de l'augmentation des risques littoraux associée à l'élévation du niveau des mers dans un contexte de changement climatique. NEUMANN et al. (2015) ont ainsi montré qu'à l'échelle mondiale, le nombre de personnes vivant dans des zones basses du littoral 


\section{Thème 1 - Hydrodynamique côtière}

augmenterait de $100 \%$ ou plus d'ici à 2060. Face au défi majeur que représente le développement durable des zones littorales, une meilleure connaissance des processus physiques qui contrôlent la dynamique hydro-sédimentaire, les aléas et les évolutions morphologiques de ces environnements apparait donc indispensable.

Au cours des deux dernières décennies, l'étude de la physique des littoraux a connu un engouement intense au niveau international. Le développement d'instruments de mesure autonomes et moins coûteux, la mise en place de réseaux de mesures pérennes (e.g. réseaux CANDHIS et REFMAR en France) et la démocratisation des modèles morphodynamiques ont permis des progrès importants concernant la compréhension des mécanismes gouvernant la dynamique littorale, en particulier lorsque l'énergie des vagues est faible. A l'inverse, la dynamique des environnements dominés par les vagues est moins bien comprise, notamment lors des tempêtes où les observations in situ près de la côte font cruellement défaut. En outre, en l'absence de telles observations, les capacités prédictives des modèles sont mal connues. La dynamique associée aux ondes infragravitaires, voir BERTIN et al. (2018) pour une synthèse récente, est également mal connue lors des évènements extrêmes, alors même qu'elles contribuent de façon importante aux submersions marines. Dans ce contexte, notre étude montre tout d'abord comment une campagne de mesure réalisée au niveau d'une plage dissipative en présence de vagues de tempête a permis d'identifier des lacunes importantes dans les modèles de déferlement utilisés couramment avec des paramètres par défaut par la communauté scientifique. Ces données montrent ensuite que la présence de zones de déferlement kilométriques permet le développement d'ondes IG à très basse fréquence $(\mathrm{O}(0.005 \mathrm{~Hz}))$, dont le comportement n'avait jamais été décrit auparavant pour ce type d'environnement. Nous concluons en discutant de l'importance des observations en présence de vagues de tempête.

\section{Description de la zone d'étude}

Notre zone d'étude correspond à la plage de St-Trojan, située au sud-ouest de l'Ile d'Oléron et se développe sur une flèche sableuse de $8 \mathrm{~km}$, limitée au sud par l'embouchure de Maumusson (figure 1). La zone pré-littorale présente une pente très faible, l'isobathe $20 \mathrm{~m}$ étant située à environ $10 \mathrm{~km}$ du trait de côte. Le régime de marée est macro-tidal et semi-diurne, avec un marnage atteignant $5.5 \mathrm{~m}$. Le régime d'agitation est énergétique, avec une hauteur significative $\left(H_{s}\right)$ moyenne de $1.60 \mathrm{~m}$ à $30 \mathrm{~m}$ de fond et une direction ouest à nord-ouest (DODET et al., 2019). Lors des tempêtes, les $H_{s}$ au large peuvent atteindre voire dépasser $10 \mathrm{~m}$ avec des périodes de pic $T_{p}$ supérieures à $20 \mathrm{~s}$ (GUÉRIN et al., 2018). Cette plage est composée de sables fins $\left(d_{50} \sim 0.2 \mathrm{~mm}\right)$ et présente une morphologie dissipative, avec des pentes de l'ordre de 1:100 en bas d'estran, atteignant 1:25 en haut d'estran lorsqu'une berme se développe après la persistance de vagues de faible énergie (GUÉRIN et al., 2018). 


\section{XVI'̀mes Journées Nationales Génie Côtier - Génie Civil \\ Le Havre 2020}
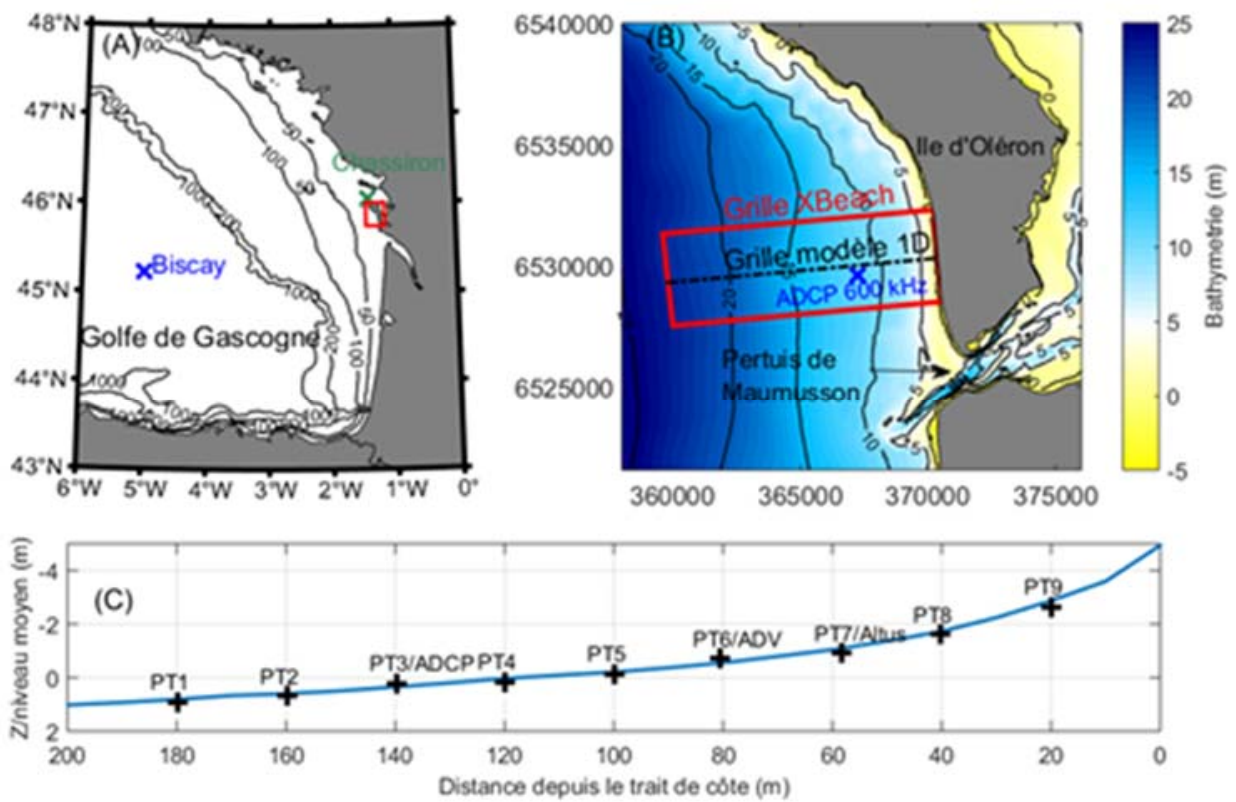

Figure 1. (A) Localisation de la zone d'étude dans le Golfe de Gascogne, (B) bathymétrie détaillée de la zone d'étude, montrant la position de l'ADCP $600 \mathrm{kHz}$ et l'extension des grilles de calcul et (C) profil de la zone intertidale avec position des capteurs déployés lors de la campagne de février 2017.

\section{Méthodes et données}

\subsection{Description de la campagne de mesure et traitement des données}

Une campagne de mesure a été réalisée début février 2017, afin de mesurer l'hydrodynamique et les vagues associées à la tempête Kurt (03/02/2017), qui ont atteint des $H_{s}=9.5 \mathrm{~m}$ et des $T_{p}=22 \mathrm{~s}$ au niveau de la bouée Biscay (figure 1-A). Un profileur de courant ADCP équipé d'un capteur de pression a été déployé par $10 \mathrm{~m}$ de fond au large de la plage étudiée du 30/01/2017 au 09/02/2017 (figure 1-B). 9 capteurs de pression, un courantomètre $\mathrm{ADCP}$ à haute résolution et un courantomètre $\mathrm{ADV}$ ont été déployés tous les $20 \mathrm{~m}$ le long d'un transect perpendiculaire à la zone intertidale du 01/02/2017 au 03/02/2017. Ces deux capteurs ont notamment été utilisés pour séparer les ondes IG incidentes et réfléchies selon la méthode de GUZA et al. (1984). Les mesures de pression au fond ont été corrigées de la pression atmosphérique mesurée à Chassiron (figure 1-A) et des échantillons de 30 minutes ont été analysés. Des spectres de densité d'énergie $E_{p}(f)$ ont été calculés à l'aide de transformées de Fourier, en utilisant 19 sous-blocs avec un fenêtrage de Hanning ( 38 degrés de liberté). Ces spectres de pression ont été transformés en spectres d'élévation de la surface libre $E(f)$ en appliquant une fonction de transfert dérivée de la théorie linéaire. La hauteur spectrale $H_{m 0}$ a été calculée comme suit :

$H_{m 0}=4 \sqrt{m_{0}}$ 


\section{Thème 1 - Hydrodynamique côtière}

où mo est le moment d'ordre 0 défini par :

$m_{0}=\int_{f \min }^{f \max } E(f) d f$

Pour la hauteur $H_{m 0}$ dans la bande gravitaire, $f_{\max }$ est fixé à $0.4 \mathrm{~Hz}$ et $f_{\min }$ correspond à la fréquence de coupure entre les bandes gravitaire et IG, définie comme la moitié de la fréquence de pic des vagues incidentes d'après HAMM \& PERONNARD (1997).

\subsection{Modélisation 1D de la dissipation des vagues}

Afin d'étudier la propagation et la dissipation des vagues entre la zone pré-littorale et le rivage, nous avons appliqué le modèle 1D de BATTJES \& JANSSEN (1978), qui suppose un équilibre entre la conservation du flux d'énergie des vagues et leur dissipation par déferlement bathymétrique et par frottement au fond :

$$
\frac{\partial E \cdot C_{g}}{\partial x}=-\varepsilon_{\text {break }}-\varepsilon_{\text {bfric }}
$$

Dans cette équation, $C_{g}$ est la vitesse de groupe des vagues calculée à partir de la théorie linéaire et $E$ est l'énergie totale $\left(1 / 16 \rho g H_{s}^{2}\right)$. Ebfric correspond au terme de dissipation par frottement au fond et a été utilisé avec des paramètres par défaut et Ebreak correspond à la quantité d'énergie dissipée par déferlement:

$\varepsilon_{\text {break }}=\frac{\alpha}{4} \rho g Q_{b} f_{m 01} H_{m}^{2}$

Dans l'équation (4), $f_{m 01}$ est la fréquence moyenne des vagues, $Q_{b}$ est la fraction de vagues déferlées, $H_{m}$ est la hauteur maximale des vagues, définie comme la hauteur d'eau locale multipliée par l'indice de déferlement $\gamma$. Le paramètre $\alpha$ est un coefficient de proportionnalité du taux de dissipation que les auteurs proposent de fixer à 1 . Cependant, dans une étude récente, PEZERAT et al. (2020) suggèrent que $\alpha$ peut être estimé comme 40 fois la pente locale du fond, en accord avec l'étude pionnière de LE MEHAUTE (1962).

\subsection{Modélisation numérique des ondes IG à l'aide d'XBeach}

Afin de compléter l'analyse des données expérimentales, nous avons implémenté le système de modélisation XBeach (ROELVINK et al., 2009) en 2DH sur la zone d'étude. L'espace géographique est discrétisé selon une grille rectilinéaire s'étendant sur $5 \mathrm{~km}$ dans la direction nord-sud et entre le trait de côte jusqu'à des profondeurs de $27 \mathrm{~m}$ dans la direction est-ouest (figure 1B), avec un pas en espace variant de $15 \mathrm{~m}$ au large à $1 \mathrm{~m}$ en haut de plage. XBeach est forcé avec des séries temporelles de hauteurs d'eau mesurées au niveau de l'ADCP $600 \mathrm{kHz}$ et de spectres directionnels calculés à partir d'une application régionale de WaveWatchIII décrite dans GUÉRIN et al. (2018). 


\section{XVİ̀mes Journées Nationales Génie Côtier - Génie Civil \\ Le Havre 2020}

\section{Résultats et discussion}

\subsection{Dissipation des vagues de tempête}

L'analyse des données expérimentales montre que les vagues au niveau de l'ADCP 600 $\mathrm{kHz}$ sont caractérisées par des $H_{m 0}$ de $2.0 \mathrm{~m}$ et des $T_{p}$ de l'ordre de 13 à $14 \mathrm{~s}$ au début de la campagne et ont atteint $5.8 \mathrm{~m}$ et $22 \mathrm{~s}$ lors du passage de la tempête Kurt.
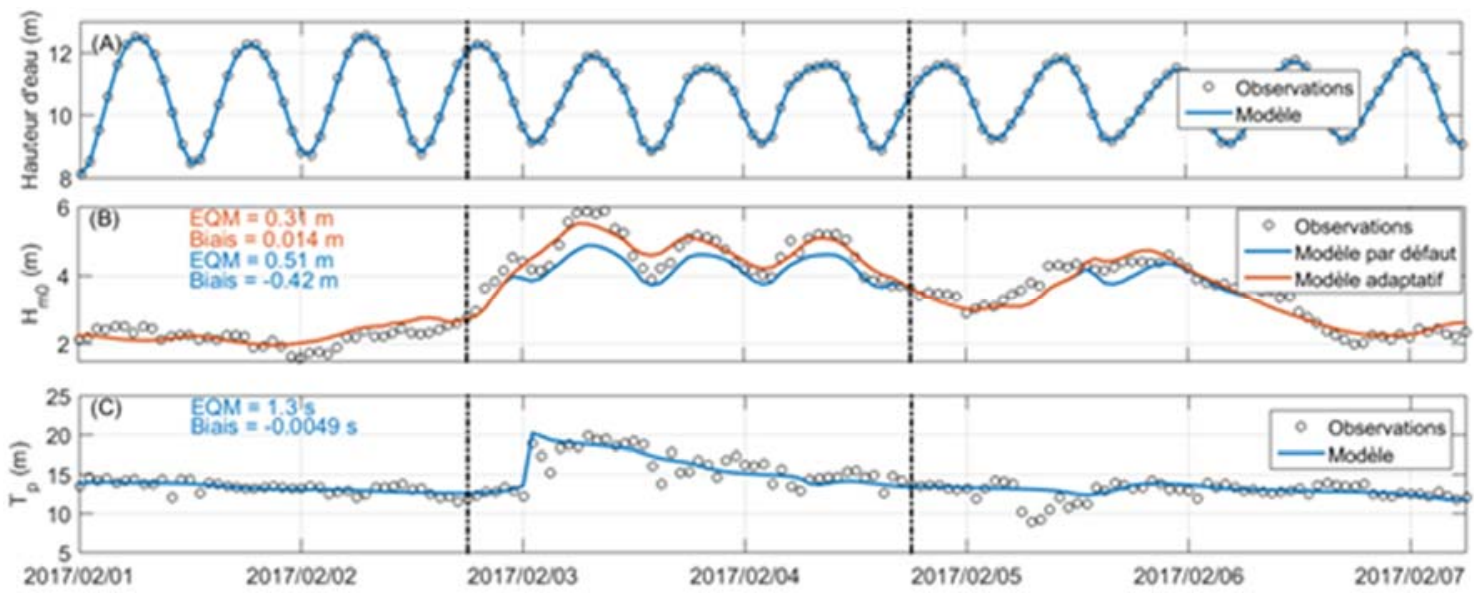

Figure 2. (A) Hauteurs d'eau, (B) hauteur $\mathrm{HmO}$ et (C) période de pic Tp des vagues observées (cercles noirs) et simulées à l'aide du modèle de BATTJES \& JANSSEN (1978) avec des paramètres de déferlement par défaut (bleu) et adaptatifs (orange). Les erreurs statistiques sont calculées pour la période de la tempête Kurt, matérialisée par les deux lignes pointillées verticales.

Ces paramètres moyens sont bien reproduits avec le modèle de BATTJES \& JANSSEN (1978) en dehors du passage de la tempête Kurt. Au pic de la tempête, les $\mathrm{H}_{\mathrm{m} 0}$ sont fortement sous-estimées avec la paramétrisations par défaut, avec une erreur quadratique moyenne (EQM) dépassant $0.5 \mathrm{~m}$, du fait d'un biais négatif dépassant temporairement $1.0 \mathrm{~m}$ (figure 2B). De surcroit, la valeur de $\gamma$ à 0.73 est trop forte pour la zone intertidale, où BERTIN et al. (2008) ont montré qu'une valeur de 0.65 permettait d'améliorer sensiblement les prédictions. Notre paramétrisation adaptative du déferlement permet d'améliorer les prédictions de façon substantielle, en éliminant le biais négatif, ce qui réduit l'erreur RMS de $40 \%$. L'analyse spatiale des résultats du modèle par défaut au pic de la tempête montre qu'il aboutit à une dissipation des vagues non nulle dès le début du profil modélisé ( $27 \mathrm{~m}$ de profondeur). Bien que les taux de dissipation soient faibles à de telles profondeurs, l'intégration de cette dissipation sur plusieurs $\mathrm{km}$ explique directement les biais négatifs observés avec le modèle par défaut et la correction de ce problème avec notre paramétrisation adaptative. 


\section{Thème 1 - Hydrodynamique côtière}

\subsection{Génération d'ondes IG à très basse fréquence}

L'analyse des observations in situ au niveau de la zone intertidale montre le développement d'ondes IG de hauteur $H_{m 0}$ dépassant $1.5 \mathrm{~m}$ et dont la période moyenne de la composante incidente $T_{m 02, I G+}$ augmente de 50 à $100 \%$ au pic de la tempête Kurt (figure $3 \mathrm{~B}$ et $\mathrm{C}$ ). Cette évolution est raisonnablement reproduite par XBeach, avec une EQM de l'ordre de $12 \%$.
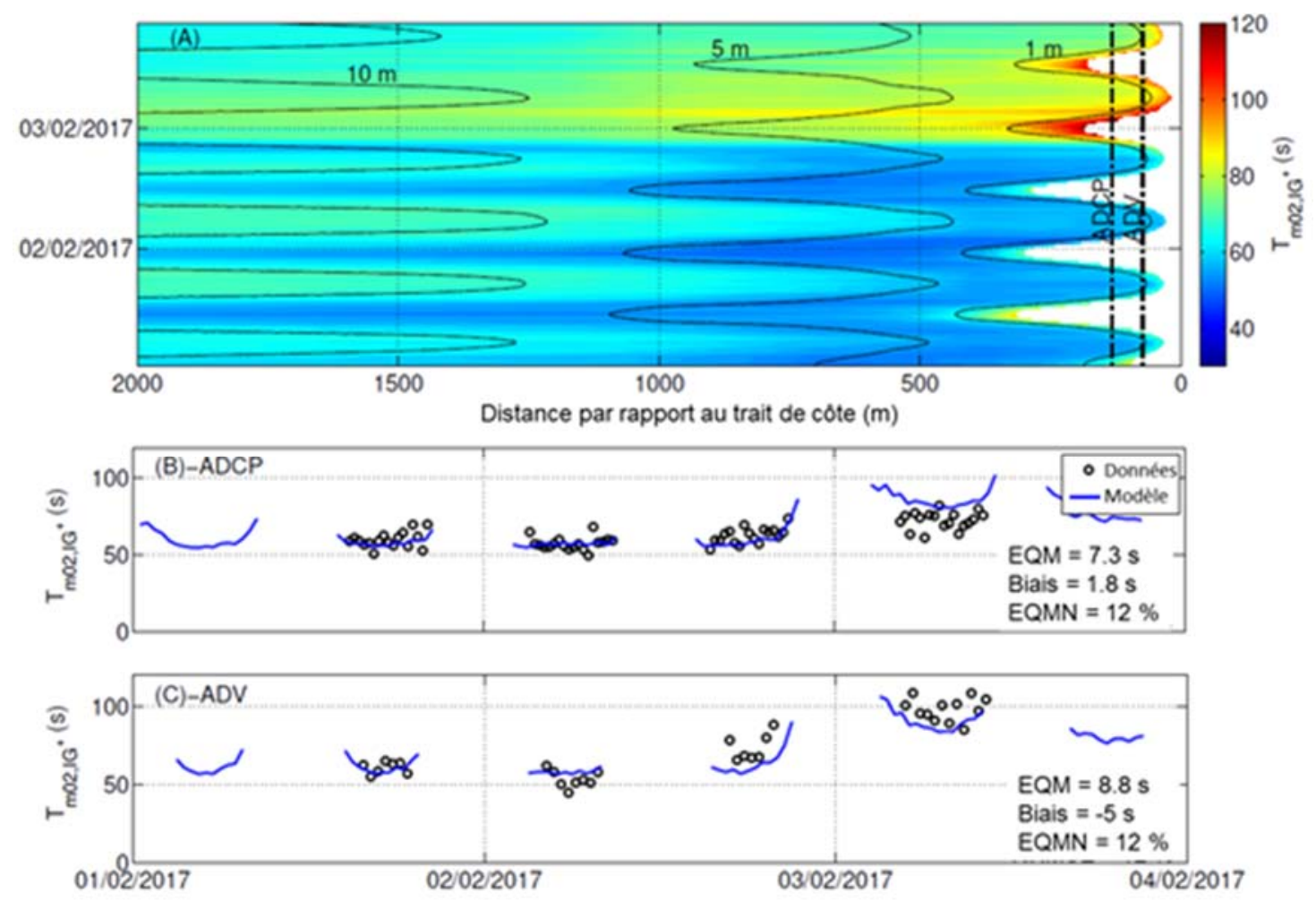

Figure 3. (A) Evolution spatio-temporelle de la période des ondes IG incidentes Tm02,

IG+ calculée à l'aide des résultats de XBeach et comparaison entre Tm02, IG+ calculée à partir des observations et des résultats numériques au niveau de l'ADCP (B) et de l'ADV $(C)$.

Dans le détail, l'évolution spatio-temporelle (figure 3A) et les séries temporelles de ce paramètre au niveau des deux courantomètres ADCP et ADV (figure $3 \mathrm{~B}$ et $\mathrm{C}$ ) montrent que $T_{m 02, I G+}$ augmente d'environ $20 \%$ près du rivage, lorsque les hauteurs d'eau sont inférieures à $1 \mathrm{~m}$. Ce comportement est expliqué par la dissipation par déferlement de la partie haute fréquence du spectre des ondes IG. Ce mécanisme ne peut par contre pas expliquer la forte augmentation de $T_{m 02, I G+}$ lors de la tempête Kurt pour des hauteurs d'eau de 5 à $10 \mathrm{~m}$ (figure 3A). L'hypothèse de transferts d'énergie vers les basses fréquences par interaction entre triplets de fréquence a été testée par analyse bi spectrale selon la méthode stochastique de HERBERS et al. (2000). Cette analyse montre que les transferts 


\section{XVİ̀mes Journées Nationales Génie Côtier - Génie Civil \\ Le Havre 2020}

d'énergie s'effectuent essentiellement vers des harmoniques supérieures, ce qui explique par ailleurs la modulation tidale de $T_{m 02, I G+}$ au cours des trois cycles de marée précédent la tempête Kurt (figure 3A).
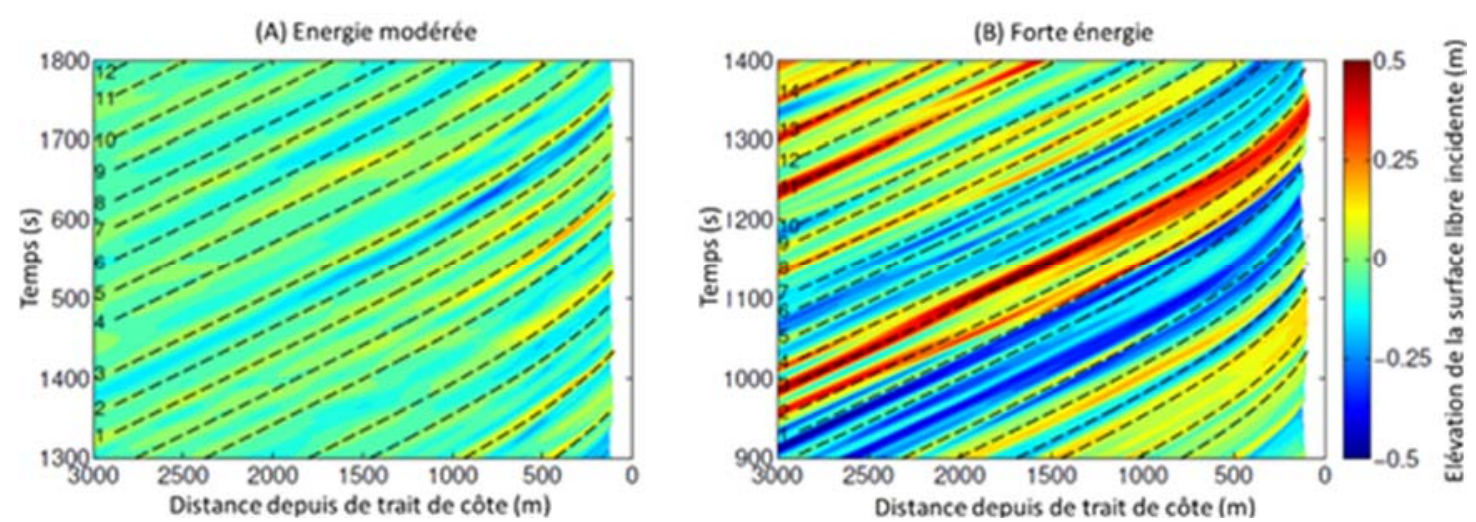

Figure 4. Evolution spatio-temporelle de la surface libre incidente en conditions

d'énergie modérée (A) et en condition de forte énergie (B). Les lignes pointillées correspondent aux creux des groupes de vagues et permettent d'identifier les ondes IG qui sont liées et celles qui se propagent librement.

La figure 4 montre la surface libre des ondes IG incidentes pour des conditions d'énergie modérée et pour le pic de la tempête Kurt. Pour des conditions d'énergie modérée, les crêtes des ondes IG sont superposées aux creux des groupes de vagues le long du profil de plage, ce qui indique qu'elles se propagent à la vitesse de groupe des vagues et suggère qu'elles sont générées par le mécanisme d'onde liée (bound wave) (figure 4A). Pour des conditions de forte énergie (figure 4B), plusieurs crêtes d'ondes IG se propagent plus rapidement que le groupe de vague (e.g. crête 2 à $X=2000 \mathrm{~m}$ et crête 4 à $X=2500 \mathrm{~m}$ ) ce qui traduit leur libération dans la zone de déferlement. La largeur kilométrique de la zone de déferlement permet l'appariement de ces ondes libres avec des ondes liées, ce qui entraine une diminution du nombre d'ondes IG et explique l'augmentation de leur période moyenne à l'approche du rivage.

\section{Conclusions et perspectives}

Une campagne de mesure a été réalisée au SO de l'Ile d'Oléron lors de la tempête Kurt, qui a entrainé le développement de zones de déferlement de plus de $3 \mathrm{~km}$ de large avec des $H_{m 0}$ approchant $6 \mathrm{~m}$. L'application du modèle 1D de BATTJES \& JANSSEN (1978) avec des paramètres par défaut a tout d'abord montré de forts biais négatifs au pic de la tempête, qui peuvent être aisément corrigés en utilisant une paramétrisation adaptative. Les principaux modèles de vague opérationnels (e.g. PREMIVER, Météo France) utilisant le modèle de BATTJES \& JANSSEN (1978), il est probable qu'ils sous-estiment la hauteur des vagues de tempête en zone littorale, ce qu'il conviendra de vérifier au 


\section{Thème 1 - Hydrodynamique côtière}

niveau d'autres sites d'étude. Cette même campagne de mesure a également permis de mettre en évidence la génération de fortes ondes $\mathrm{IG}$, dont la période augmente à l'approche du rivage. Ce comportement a été expliqué par le déferlement des ondes IG les plus courtes en eau peu profonde, mais aussi par l'appariement d'ondes libres et liées se propageant à des célérités légèrement différentes au travers de zones de déferlement kilométriques. Ce dernier mécanisme inédit à notre connaissance a des conséquences importantes car les ondes IG très longues ainsi générées peuvent subir une forte réflexion même au niveau d'une plage dissipative ou entrainer des submersions marines dans les zones basses du littoral. Les deux exemples présentés dans cette étude soulignent l'importance de réaliser des campagnes de mesure en présence de vagues de tempête, afin de mieux comprendre et prédire les aléas littoraux, qui seront amenés à s'intensifier en raison du changement climatique.

\section{Remerciements}

Ces travaux de recherche ont été menés dans du programme de Chaire Régionale EVEX. KM remercie le soutien venant l'Université de Bordeaux, au travers d'une bourse postdoctorale internationale Idex (nb. 1024R-5030). M.P. est cofinancé par la CDA de La Rochelle et le projet FEDER DURALIT. B.M. est financé par le projet Segundo Torrão dans le cadre du programme « Nouveaux Commanditaires - Sciences » de la Fondation de France. LL est financée par la Région Nouvelle Aquitaine et l'UNIMA. L'équipe développant les modèles XBeach et WWIII est chaleureusement remerciée.

\section{Références bibliographiques}

BATTJES J.A., JANSSEN J.P.F.M. (1978). Energy loss and set-up due to breaking of random waves. Proc. $16^{\text {th }}$ Int. Conf. Coastal Engineering, ASCE, pp 569-587. https://doi.org/10.1061/9780872621909.034

BERTIN X., CASTELlE B., CHAUMILlON E., BUTEL R., QUIQUE, R, (2008). Estimation and inter-annual variability of the longshore transport at a high-energy dissipative beach: the St Trojan beach, SW Oléron Island, France. Continental Shelf Research, Vol. 28(10-1), pp 1316-1332. https://doi.org/10.1016/j.csr.2008.03.005

BERTIN X., DE BAKKER A., VAN DONGEREN A., COCO G., ANDRÉ G., ARDHUIN F., BONNETON P., BOUCHETTE F., CASTELLE B., CRAWFORD W.C., DAVIDSON M., DEEN M., DODET G., GUÉRIN T., INCH K., LECKLER F., MCCALL R., MULLER H., OLABARRIETA M., ROELVINK D., RUESSINK G., SOUS D., STUTZMANN E., TISSIER M., (2018). Infragravity waves: From driving mechanisms to impacts. Earth-Science Reviews, Vol. 177, pp 774-799. https://doi.org/10.1016/j.earscirev.2018.01.002 


\section{XVIèmes Journées Nationales Génie Côtier - Génie Civil \\ Le Havre 2020}

DODET G., BERTIN X., BOUCHETTE F., GRAVELlE M., TESTUT L., WÖPPELMANN G. (2019). Characterization of sea-level variations along the metropolitan coasts of France: waves, tides, storm surges and long-term changes. Journal of Coastal Research, Special Issue No. 88, pp 10-24. https://doi.org/10.2112/SI88-003.1

GUÉRIN T., BERTIN X., COULOMBIER T., DE BAKKER A., (2018). Impacts of wave-induced circulation in the surf zone on wave setup. Ocean Modelling, Vol. 123, pp 86-97. https://doi.org/10.1016/j.ocemod.2018.01.006

GUZA R.T., THORNTON E.B., HOLMAN R.A., 1984. Swash on steep and shallow beaches. Coastal Engineering Proceedings, 1(19), 48. https://doi.org/10.9753/icce.v19.48

HAMM L., PERONNARD C., (1997). Wave parameters in the nearshore: A clarification. Coastal Engineering, Vol. 32, Issues 2-3, pp 119-135. https://doi.org/10.1016/S0378-3839(97)81746-2

HERBERS T. H.C., RUSSNOGLE N.R., ELGAR S. (2000). Spectral energy balance of breaking waves within the surf zone. Journal of Physical Oceanography, Vol. 30, pp 2723-2737. https://doi.org/10.1175/1520-0485(2000)030<2723:SEBOBW >2.0.CO;2

PEZERAT M., MARTINS K., BERTIN X. (2020). Modelling storm waves in the nearshore area using spectral models. Journal of Coastal Research, Special Issue No. 95, pp 1-5. In press

LE MEHAUTE B., (1962). On non-saturated breakers and the wave run-up. Proceedings of $8^{\text {th }}$ Conference on Coastal Engineering, Mexico City, Mexico, Issue $n^{\circ} 8, p p$ 77-92, https://doi.org/10.9753/icce.v8.6

NEUMANN B., VAFEIDIS A.T., ZIMMERMANN J., NICHOLLS R.J. (2015). Future coastal population growth and exposure to sea-level rise and coastal flooding - a global assessment, PLOS ONE 10(6): e0131375. https://doi.org/10.1371/journal.pone.0118571

ROELVINK D., RENIERS A., VAN DONGEREN A., VAN THIEL DE VRIES J., MCCALL R., LESCINSKI J. (2009). Modelling storm impacts on beaches, dunes and barrier islands. Coastal Engineering Vol. 56, Issues 11-12, pp 1133-1152. https://doi.org/10.1016/j.coastaleng.2009.08.006 
Thème 1 - Hydrodynamique côtière 